\title{
EL SISTEMA PRONOMINAL ÁTONO DE TERCERA PERSONA DE OBJETO DIRECTO EN EL ESPAÑOL HABLADO POR GUAYMÍES COSTARRICENSES
}

Rebeca Marín Esquivel

\section{(9) $\odot \Theta \Theta$}

Esta obra está bajo una licencia Creative Commons

Reconocimiento-No Comercial-Sin Obra Derivada 



\title{
EL SISTEMA PRONOMINAL ÁTONO DE TERCERA PERSONA DE OBJETO DIRECTO EN EL ESPAÑOL HABLADO POR GUAYMÍES COSTARRICENSES
}

\section{THE THIRD-PERSON PRONOMINAL SYSTEM OF DIRECT OBJECT IN SPANISH SPOKEN BY COSTA RICAN GUAYMÍES}

\author{
Rebeca Marín Esquivel
}

\begin{abstract}
RESUMEN
En el presente artículo se describe la organización del sistema pronominal átono de tercera persona de objeto directo expresado en el habla de seis individuos guaymíes costarricenses. Primero, se cuantifica la aparición de pronombres átonos de tercera persona, tomando en cuenta su relación de discordancia y concordancia con los sintagmas correferenciales. Segundo, se explica el influjo que podría estar teniendo el guaymí como lengua de contacto.

Palabras clave: guaymí, sistema, pronombres, bilingüismo, contacto.
\end{abstract}

\begin{abstract}
In this article we describe the organization of the third person pronominal pronominal system of direct object expressed in the speech of six Guaymi Costa Rican individuals. First, the occurrence of unstressed third-person pronouns is quantified, taking into account their relationship of discordance and concordance with the co-referential phrases. Second, it explains the influence that guaymí, as a contact language, could have in Spanish.

Key words: guaymi, system, pronouns, bilingualism, contact.
\end{abstract}

\section{Introducción}

Actualmente se pueden destacar situaciones de bilingüismo social en la región hispanoamericana, tanto en Mesoamérica y en el Área Intermedia Centroamericana (la Baja Centroamérica incluida en esta última), como en las áreas Caribeña, Peruana y Amazónica que albergan las variedades del español hablado por nativos de la Amazonia o de la Guajira, además

\footnotetext{
M. L. Rebeca Marín Esquivel. Universidad Nacional de Costa Rica. Facultad de Filosofía y Letras. Profesora de Lingüística.

Correo electrónico: rebekme@gmail.com
}

Recepción: 17- 03- 17

Aceptación: 29- 03- 17 
del español hablado en las regiones andinas y mapuches, entre muchas otras (ConstenlaUmaña, 1991; Palacios, 2008; Quesada-Pacheco, 2008a). Este panorama multilingüístico sirve de contexto para la mutua interacción entre el español y las lenguas amerindias. Desde este punto de vista, es valioso rescatar la influencia, poca o mucha, directa o indirecta, que han tenido estas lenguas sobre las variedades del español hablado en Hispanoamérica (Aleza y Enguita, 2010). Los rasgos del español hispanoamericano en situación de contacto con lenguas indígenas dependen de muchos factores sociolingüísticos, dentro de los cuales podemos mencionar la intensidad del contacto entre la comunidad indígena e hispanohablante, el tipo de bilingüismo individual y la permanencia que un rasgo o patrón lingüístico tenga en una variedad con contigüidad histórica (Palacios-Alcaine, 2002, 2005 y 2011).

Los estudios desarrollados en Costa Rica sobre contacto lingüístico abordan temas como la influencia discursiva, léxica, fonética y gramatical de las lenguas vernáculas en el español (Lininger-Ross, 1991; Constenla-Umaña, 2002; Sánchez-Avendaño, 2013; QuesadaPacheco, 2008 y 2009) ${ }^{1}$, y el desplazamiento de las lenguas en peligro (Portilla-Chaves, 1986; Sánchez-Avendaño, 2012). En particular, Constenla-Umaña (2002) y Quesada-Pacheco (2008) indican que la influencia de las lenguas indígenas en las distintas variedades de español costarricense no está descontextualizada de la situación de bilingüismo que se extiende a lo largo y ancho de toda América. Esta situación se caracteriza por tener al español como lengua dominante sobre las lenguas indígenas pero, además, por mostrar fenómenos muy típicos en circunstancias de bilingüismo social, por ejemplo, el desplazamiento de las lenguas.

No obstante, aunque las lenguas indígenas coexisten con el español hablado en nuestro país, las investigaciones desarrolladas por la lingüística costarricense en esta línea siguen siendo aún muy escasas. Con excepción de los estudios realizados por Lininger-Ross (1991) y Sánchez-Avendaño (2015) que describen rasgos lingüísticos del español en contacto con las lenguas bribri y malecu respectivamente, uno de los vacíos que se deja entrever es la falta de más descripciones sobre variedades del español circunscritas a distintas situaciones de bilingüismo social en zonas indígenas. Tan solo se ha mencionado superficialmente que el español en contacto con lenguas indígenas de la familia chibcha presenta rasgos fonéticos, morfosintácticos, discursivos, etc. (Quesada-Pacheco, 2008a y 2009) debido al influjo de estas lenguas. Tampoco se ha publicado ningún estudio del español hablado por guaymíes costarricenses que contemple, ya sea una descripción de todos los niveles lingüísticos (fonético, morfológico, sintáctico, etc.), o bien un análisis pormenorizado de algún fenómeno particular.

Para el sistema pronominal átono de tercera persona de objeto directo, se han registrado al menos dos fenómenos generales sobre contactos lingüísticos en Hispanoamérica, principalmente, en estudios sobre el español en contacto con las lenguas guaraní, maya, quechua, entre otras: la reestructuración del sistema de clíticos que conduce a la simplificación de este a favor de $l o$, especificando género y caso (y algunas veces el número), o un tipo de simplificación a favor de $l e(s)$ (Palacios, 2007, 2008). Además, en estos fenómenos actúa el mecanismo de convergencia lingüística (ver apartado 1.1) como detonador de los principales cambios inducidos por contacto (Palacios-Alcaine, 2007, 2008 y 2011).

Así las cosas, en el presente artículo se describe la organización del sistema pronominal átono de tercera persona de objeto directo expresado en el habla de seis individuos guaymíes costarricenses. Primero, se cuantifica la aparición de estos pronombres, tomando en cuenta su relación de discordancia y concordancia con los sintagmas correferenciales, y posteriormente, 
se explica el influjo que podría estar teniendo el guaymí como lengua de contacto en la organización de sistema.

Para simplificar la redacción, en todo el documento se utilizará indistintamente "pronombre átono", "clítico" o solo "pronombre", y, cada vez que se mencione "sistema pronominal átono", "sistema pronominal" o "sistema", se está haciendo referencia exclusivamente a "sistema pronominal átono de tercera persona". Además, la etiqueta "español costarricense sin contacto" hace referencia al español costarricense sin contacto reciente con otra lengua, en particular con el guaymí2.

\subsection{Referentes teóricos}

La Lingüística del Contacto es el campo de investigación sobre las variaciones y cambios que se producen por el contacto de lenguas, sobre los mecanismos operantes en tales cambios, así como sobre las causas, los efectos y en los resultados estructurales, léxicos, pragmáticos y sociolingüísticos derivados de las influencias mutuas entre las lenguas (Kaufman y Thomason, 1988; Apple y Muysken, 1996; Thomason, 2001; Matras, 2009).

En esta línea de investigación, Palacios-Alcaine (2002, 2010 y 2011) elaboró un modelo dinámico del contacto de lenguas basándose en los fenómenos del español hablado por indígenas en distintos escenarios sociolingüísticos de Latinoamérica. Para comenzar, PalaciosAlcaine (2011) señala que los hablantes de regiones donde predomina el bilingüismo social, en su intento por lograr una comunicación efectiva con otros interlocutores lingüísticamente diversos, tienden a producir innovaciones morfosintácticas mediante procesos creativos. Estas innovaciones consisten en rasgos que caracterizan las variedades de los usuarios bilingües, así como las de los monolingües expuestos al input de aquellos. Este es precisamente el contacto lingüístico experimentado por los hablantes amerindios, ya sean bilingües español-lengua indígena, o monolingües en una u otra lengua.

El uso de ambas lenguas ocurre como un hecho cotidiano de los hablantes indígenas, sujeto a las eventualidades de la variación y el cambio inducido por contacto. La autora prefiere referirse a estos términos como procesos dinámicos que participan activamente en la formación y consolidación de variedades de habla. Acorde con su dinamismo, los cambios pueden ser individuales o estar extendidos a una comunidad entera de forma sistemática y permanente; más aún, ocurren en medio de la complejidad de la diversidad étnica, social y cultural que identifica a las zonas de contacto y a sus hablantes.

Los recursos sintácticos están asociados a la identidad de los grupos lingüísticos y a su cultura. En el complejo lingüístico y cultural existen semejanzas y diferencias entre los hablantes indígenas miembros de un mismo colectivo étnico que, por razones individuales, han desarrollado una competencia bilingüe distinta en español en comparación con la lengua indígena. Al conocer el tipo de bilingüismo de un hablante se pueden explicar las características sobresalientes de su modalidad de habla.

Palacios-Alcaine (2011, p. 20) explica lo anterior por medio de un continuum en el que entran las modalidades de habla de los bilingües y monolingües en situaciones de contacto entre español y lengua indígena. En el centro del continuum (Figura 1) se establecen los tipos de bilingüismo consecutivo y simétrico según la competencia que el individuo ha alcanzado en ambas lenguas: el bilingüe consecutivo tiene la lengua indígena como lengua materna y dominante, mientras que el bilingüe simétrico tiene dominio casi idéntico en las dos lenguas ${ }^{3}$ : 


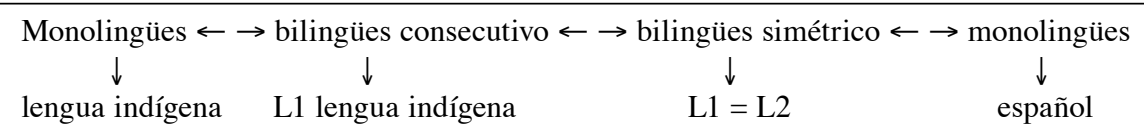

Figura 1. Modalidades de habla bilingüe. Tomado de Palacios-Alcaine (2011, p. 20)

En vista de que los fenómenos de contacto lingüístico constituyen procesos dinámicos, Palacios-Alcaine (2011) señala que los cambios inducidos por contacto conocidos como cambios indirectos están motivados por factores internos y externos a la lengua. Los primeros corresponden a cambios ocurridos por la evolución interna del sistema lingüístico, mientras que los segundos manifiestan la generación de cambios producidos por entrar en contacto con otras lenguas. La variación que aparentemente se relaciona con un cambio existe en la lengua misma y no corresponde a ningún elemento funcional o léxico de otra lengua. Según PalaciosAlcaine (2011), la definición de este tipo de cambios es la siguiente:

\begin{abstract}
Entiendo los cambios indirectos [...] como cambios multicausales compatibles con las tendencias internas del sistema [...] No supone importación de material ajeno, ya que el cambio lingüístico se produce a partir de una variación ya existente en la lengua. Así, mediante la influencia indirecta de una lengua en contacto A surgen variaciones gramaticales muy significativas, generalmente en el registro oral coloquial de la lengua B, que aprovechan la propia evolución interna de esa lengua B para hacer aflorar estrategias gramaticales cuya funcionalidad comunicativa obedece a procesos cognitivos de la lengua A de contacto (Palacios-Alcaine, 2011, pp. 25-26)
\end{abstract}

Comúnmente, se producen varios efectos lingüísticos, como la aceleración de procesos de cambios en curso, expansión de los resultados de dichos cambios, la reestructuración de los sistemas y la reasignación de nuevas funciones y significados a las estructuras de la lengua. El mecanismo de convergencia lingüística constituye un denotador de los diferentes cambios indirectos que aquí se describen: “[...] los fenómenos de convergencia lingüística se caracterizan como un conjunto de procesos paralelos que desembocarán en el desarrollo de estructuras gramaticales comunes en las lenguas de contacto [...]” (Palacios-Alcaine, 2004, p. 84).

Los rasgos estructurales comunes entre el español y las lenguas de contacto estudiadas por Palacios-Alcaine (2004 y 2011) son: a) variaciones en el sistema pronominal átono del español sin contacto con otras lenguas que implican los rasgos de caso y género (laísmo, leísmo y loísmo), y b) estas lenguas amerindias con las que el español entra en contacto no gramaticalizan género o número obligatoriamente, el caso morfológico no presenta correspondencia directa con el acusativo en el español, y tampoco poseen un sistema de clíticos similar al español (Palacios-Alcaine, 2004). Los rasgos inestables del español (género, número y caso) se aproximan a los de la lengua de contacto pues la primera es influida por la segunda. La principal consecuencia estructural de que los rasgos converjan es su neutralización y posterior simplificación del paradigma pronominal.

\title{
1.2 Metodología
}

El tipo de hablantes que pueden integrar la muestra poblacional en la investigación lingüística no siempre resulta predeterminable desde un inicio; más bien, esta decisión puede ser tomada a la luz de las primeras observaciones de campo (Martín-Crespo y Salamanca-Castro, 2007). El acceso a un primer hablante nos puso en contacto con otros a los que se les preguntó si deseaban participar en nuestro estudio (muestreo en cadena). Esta selección de individuos hecha a partir del muestreo en cadena abrió, consecuentemente, la 
posibilidad de hacer una selección por muestreo "teórico" o "deliberado" (Martín-Crespo y Salamanca-Castro, 2007), o dicho en otras palabras, "intencionado" o "predeterminado" (Silva-Corvalán, 2001).

Una cantidad de doce hablantes constituyó el tope o número máximo del muestreo en cadena, debido al tiempo que la investigadora necesitó para reunir y convencer a los individuos del estudio. Así, cuando tuvimos un estimado de doce candidatos por medio del muestreo en cadena, se seleccionaron seis hablantes masculinos (bilingües guaymí-español) de los cuales dos se ubican en cada uno de los siguientes tres rangos etarios: de 50 a 60 años (llamados de ahora en adelante $\mathrm{H} 1$ y H2), de 35 a 45 (H3 y H4) y de 20 a 30 años (H5 y H6). Conforme la explicación del análisis, se hará referencia al tipo de bilingüismo de cada uno de los entrevistados ${ }^{4}$.

Además, sumamos un hablante hispanocostarricense de control, residente de Sabanilla, lugar ubicado a unos 7 kilómetros de La Casona (llamado de ahora en adelante HHC) ${ }^{5}$, cuyas muestras orales se han comparado con las de los guaymíes. Este hablante es de sexo masculino, agricultor, con primaria incompleta, y tiene sesenta años, edad en la cual las personas poseen mucha seguridad lingüística y tienden a ser menos influidas por los diversos factores del medio social (Moreno-Fernández, 1998 y Silva-Corvalán, 2001).

En cuanto a la técnica de recolección de datos, se utilizaron dos entrevistas semiestructuradas; una, en la que los individuos compartieron información sobre sus vidas personales ${ }^{6}$, y otra, por medio de la cual se recogieron textos orales sobre temáticas libres. La duración de ambas entrevistas fue de un tiempo estimado de 40 minutos para cada individuo (guaymí e hispanocostarricense).

El siguiente paso fue organizar el corpus para describir los datos lingüísticos pertinentes en nuestro estudio. Así las cosas, fueron identificados y clasificados todos los ejemplos de cláusulas con pronombre átonos de tercera persona de objeto directo según las propiedades gramaticales de género y número. Esta clasificación de ejemplos constituyó el punto de partida para la siguiente parte del análisis, en la cual se realizó una cuantificación de los fenómenos encontrados, pues, para propósitos de este trabajo, resultó conveniente identificar la cantidad de discordancias vs. concordancias excluyendo aquellos ejemplos cuyos referentes fueran ambiguos. Además, se registró la ausencia de clíticos en (al menos) dos posiciones estructurales obligatorias según la norma del español sin contacto de lenguas: en complementos con sintagmas nominales o pronombres personales tónicos introducidos por $a$ (1) y en construcciones dislocadas a la izquierda (2) (RAE, 2010) ${ }^{7}$ :

\section{La vieron a ellal *Vieron a ella}

\section{El periódico lo compra mi hijo/ *El periódico compra mi hijo}

La RAE (2010) también documenta posiciones requeridas para identificar un tipo específico de entidad o acción (31):

\section{El que desee fumar, puede hacerlo/*El que desee fumar, puede hacer}

El ejemplo muestra que para referirse al sintagma correferencial fumar, debe haber un clítico que lo codifique, de lo contrario el mensaje queda incompleto ${ }^{8}$.

Por otro lado, el objeto de estudio que nos ocupa también requirió de una descripción cualitativa que pudiera probar aquellas posibles influencias del guaymí en el español, las cuales solo pueden ser determinadas mediante la comparación entre las propiedades de género y número de las dos lenguas en contacto. 


\section{Análisis y resultados cuantitativos}

\section{$2.1 \quad H 1$}

H1 declaró entender y hablar mejor en guaymí, por lo cual consideramos que se trata de un bilingüe consecutivo con el guaymí (L1) como lengua dominante.

\subsubsection{Conteo de pronombres átonos de objeto directo en el habla de $\mathrm{H} 2$}

Para H1, únicamente se han identificado las formas clíticas lo y la en función de objeto directo, tal y como se observa en la siguiente tabla:

Tabla 1. Apariciones de pronombres átonos de objeto directo en el habla de H1

\begin{tabular}{|c|c|c|c|c|c|}
\hline \multicolumn{3}{|c|}{$\begin{array}{c}\text { LO } \\
(31 \text { de } 32)\end{array}$} & \multicolumn{3}{|c|}{$\begin{array}{c}\text { LA } \\
(1 \text { de } 32)\end{array}$} \\
\hline & Referentes & Abs. & & Referentes & Abs. \\
\hline \multirow{4}{*}{ Disc. } & Masc. sing. & 0 & \multirow{4}{*}{ Disc. } & Masc. sing. & 0 \\
\hline & Masc. pl. & 2 & & Masc. pl. & 0 \\
\hline & Fem. sing. & 7 & & Fem. sing. & 0 \\
\hline & Fem. pl. & 2 & & Fem. pl. & 0 \\
\hline & Total & 11 & & Total & 0 \\
\hline \multirow{4}{*}{ Conc. } & Masc. sing. & 20 & \multirow{4}{*}{ Conc. } & Masc. sing. & 0 \\
\hline & Masc. pl. & 0 & & Masc. pl. & 0 \\
\hline & Fem. sing. & 0 & & Fem. sing. & 1 \\
\hline & Fem. pl. & 0 & & Fem. pl. & 0 \\
\hline & Total & 20 & & Total & 1 \\
\hline Total & & 31 & & & 1 \\
\hline
\end{tabular}

El pronombre lo ocurre en 31 ocasiones distribuidas en 20/20 concordancias con referente masculino singular y 11 discordancias, de las cuales, $2 / 2$ casos se dan con referentes masculinos plurales, $7 / 7$ casos con femeninos singulares y $2 / 2$ casos con femeninos plurales; mientras que la es utilizado tan solo en $1 / 1$ en concordancia con su referente.

Así pues, por medio de los números absolutos inferimos que la forma masculina lo es predominante frente a la forma femenina $l a$, no solo en cantidad apariciones, sino también en cantidad de discordancias (al menos en la muestras orales de este individuo). Cabe mencionar que la categoría con mayor cantidad absoluta de discordancias es la de referente femenino singular'.

4. Ya lo ofendía (al otro). Conc.

5. Un pollo ojalá de montaña lo hace en arroz seco. Conc.

6. Ponerla a germinar (la semilla). Conc.

7. Curarlo bien (a la mujer). Disc.

8. Ya ya ahorita la juventud usted lo saluda ¿mä töa ño? no contesta ${ }^{10}$. DisC.

9. Llegar hablar español es como ofenderlo (a nuestros ancianos). Disc. 
10. A ellos no lo tomaban en cuenta. Disc.

11. Traerlo para acá algunas cosas. Disc.

12. (A ellas) lo maltrata mucho. DisC.

Además, hemos identificado seis omisiones de objeto directo, de las cuales dos hacen referencia a sintagmas femeninos, entretanto los cuatro restantes se refieren a sintagmas masculinos.

13. Para matar la danta, a veces para matar $\emptyset$ (danta).

14. Toda la familia que iba a consumir $\emptyset$ (la danta).

15. La gente Ø cultiva en Honduras (el ñuture $)^{11}$.

16. Esto uno va a cosechar $\emptyset$ al monte.

17. Ellos más bien no $\emptyset$ mostraban (interés) más bien nos molestaban.

\section{$2.2 \quad \mathrm{H} 2$}

H2 declaró tener una mejor comprensión y producción en guaymí que en español; así las cosas, se trata de un bilingüe consecutivo, cuya lengua dominante es el guaymí.

\subsubsection{Conteo de pronombres átonos de objeto directo en el habla de $\mathrm{H} 2$}

En los datos relativos al habla de $\mathrm{H} 2$, solo aparece el clítico lo (en singular) cumpliendo la función de objeto directo. Observemos el dato numérico exacto en la siguiente tabla:

Tabla 2. Apariciones de pronombres átonos de objeto directo en el habla de $\mathbf{H 2}$

\begin{tabular}{|l|l|l|}
\hline \multicolumn{2}{|c|}{ LO } \\
\hline & Referentes & \multicolumn{2}{|c|}{ 48) } & Abs \\
\hline \multirow{4}{*}{ Disc. } & Masc. sing. & 0 \\
\cline { 2 - 3 } & Masc. pl. & 2 \\
\cline { 2 - 3 } & Fem. sing. & 11 \\
\cline { 2 - 3 } & Fem. pl. & 2 \\
\cline { 2 - 3 } & Múltiple & 2 \\
\cline { 2 - 3 } & Sin gén. & 0 \\
\cline { 2 - 3 } & Total & 17 \\
\hline \multirow{5}{*}{ Conc. } & Masc. sing. & 29 \\
\cline { 2 - 3 } & Masc. pl. & 0 \\
\cline { 2 - 3 } & Fem. sing. & 0 \\
\cline { 2 - 3 } & Fem. pl. & 0 \\
\cline { 2 - 3 } & Múltiple & 0 \\
\cline { 2 - 3 } & Sin gén. Sing. & 31 \\
\cline { 2 - 3 } & Total & \\
\hline
\end{tabular}

Para $\mathrm{H} 2$, se identificó únicamente el uso del pronombre lo en función de objeto directo, el cual concuerda con sus referentes en 31 de 48 ocasiones, mientras que las 
discordancias ocurren en 17 de 48. Sin embargo, aunque tenemos evidencia de que la cantidad de concordancias supera a la de discordancias, llama la atención que en la totalidad de apariciones, el objeto directo se codifique en una única forma masculina y singular.

Las discordancias se distribuyen del siguiente modo: $2 / 2$ ocurren con referentes masculinos plural; 2/2, con femeninos plurales; $2 / 2$ con referente múltiple ${ }^{12}$, y 11/11 con referentes femeninos singulares, siendo esta última la cantidad de casos más alta del conjunto.

18. Nosotros lo han visto (al sulia). ConC.

19. Lo mandaba a niño. CONC.

20. Yo lo entiendo (a alguien). CONC.

21. Principalmente la idea yo lo agarro de una palabra. Disc.

22. Yo lo sé (una historia). Disc.

23. No lo dejaba sufrir (a los chiquitos). DisC.

24. Ya lo vamos a enseñar a las hijas. Disc.

25. A mi mamá, a mi abuelo y a mi abuela yo sí lo vi. DisC.

Por otra parte, las siguientes oraciones corresponden a dos casos de omisión (de objeto directo) registrados para $\mathrm{H} 2$ :

26. Mi papá yo no $\varnothing$ vi

27. Todas esas cuestiones no podía meter $\varnothing$ más

Como se aprecia, el cero fonético hace referencia a un sintagma masculino y singular en (26), mientras que (27) corresponde a un referente femenino plural.

\section{$2.3 \quad \mathrm{H3}$}

H3 corresponde a un bilingüe consecutivo con el guaymí como L1 y español como L2.

\subsubsection{Conteo de pronombres átonos de objeto directo en el habla de H3}

En las muestras orales de $\mathrm{H} 3$, se identifica la aparición de clíticos $l o(s)$ y la en función de objeto directo. A continuación se muestra la cantidad exacta de estas apariciones:

Tabla 3. Apariciones de pronombres átonos de objeto directo en el habla de $\mathbf{H 3}$

\begin{tabular}{|c|c|c|c|c|c|c|c|c|}
\hline \multicolumn{3}{|c|}{ LOS } & \multicolumn{3}{|c|}{ LO } & \multicolumn{3}{|c|}{ LA } \\
\hline \multicolumn{3}{|c|}{$(1 \mathrm{de} 79)$} & \multicolumn{3}{|c|}{ (74 de 79) } & \multicolumn{3}{|c|}{$(4$ de 79$)$} \\
\hline & Referentes & Abs & & Referentes & Abs & & Referentes & Abs \\
\hline \multirow{6}{*}{ Disc. } & Masc. sing. & 0 & \multirow{6}{*}{ Disc. } & Masc. sing. & 0 & \multirow{6}{*}{ Disc. } & Masc. sing. & 0 \\
\hline & Masc. pl. & 0 & & Masc. pl. & 13 & & Masc. pl. & 0 \\
\hline & Fem. sing. & 0 & & Fem. sing. & 25 & & Fem. sing. & 0 \\
\hline & Fem. pl. & 1 & & Fem. pl. & 7 & & Fem. pl. & 0 \\
\hline & Múltiple & 0 & & Múltiple & 2 & & Múltiple & 0 \\
\hline & Total & 1 & & Total & 47 & & Total & 0 \\
\hline
\end{tabular}




\begin{tabular}{|c|c|c|c|c|c|c|c|c|}
\hline \multirow{6}{*}{ Conc. } & Masc. sing. & 0 & \multirow{6}{*}{ Conc. } & Masc. sing. & 27 & \multirow{6}{*}{ Conc. } & Masc. sing. & 0 \\
\hline & Masc. pl. & 0 & & Masc. pl. & 0 & & Masc. pl. & 0 \\
\hline & Fem. sing. & 0 & & Fem. sing. & 0 & & Fem. sing. & 4 \\
\hline & Fem. pl. & 0 & & Fem. pl. & 0 & & Fem. pl. & 0 \\
\hline & Múltiple & 0 & & Múltiple & 0 & & Múltiple & 0 \\
\hline & Total & 0 & & Total & 27 & & Total & 4 \\
\hline
\end{tabular}

El clítico lo ocurre en 27 casos en total, en tanto su variante plural los y su variante femenina la ocurren en 1 y 4 casos, respectivamente, lo cual demuestra que el uso de la forma lo prima sobre el uso de las formas los y la. Además, lo se manifiesta discordando con su referente en 47 casos contra 27 concordancias registradas; la concuerda con su referente en 4/4 oportunidades con referentes femeninos singulares (sin presentar realizaciones discordantes), mientras que la única aparición de los corresponde a una discordancia con un referente femenino y plural. Las 47 discordancias de lo se distribuyen en 13/13 con referentes masculinos plurales, 7/7 con referentes femeninos plurales, $2 / 2$ con referentes múltiples y 25/25 con referentes femeninos singulares. Esta última resulta ser la cantidad de discordancias más alta para dicho clítico.

28. El gigante lo enterró (al hermano). ConC.

29. No lo dejaba al gigante. CONC.

30. Y lo alzó (a la niña). DisC.

31. No lo va a botar (a los niños). Disc.

32. Lo tienen a los muris más abajo ${ }^{13}$. DisC.

33. Lo voy a soltar (al hombre y a la mujer). DISC.

34. Yo trato de entenderlo (las dos culturas). Disc.

35. No sé si usted la conoce (la planta). ConC.

36. Los recibe (a ellas). Disc.

En el discurso de H3, ocurre una omisión del clítico plural las de objeto directo (58):

(58) Pero llegó descubrirØ (las plantas exactamente para esa enfermedad).

\section{$2.4 \quad H 4$}

H4 entra en la categoría de bilingüe simétrico, es decir, como aquel bilingüe que puede entender y hablar dos lenguas, mostrando un dominio equilibrado entre ambas.

\subsubsection{Conteo de pronombres átonos de objeto directo en el habla de H4}

Para H4, se registran las formas pronominales lo y la, así como la variante plural los en función de objeto directo. En la Tabla 4, se muestra el porcentaje de aparición correspondiente a cada clítico y, además, la cantidad de usos discordantes y concordantes en cada uno: 
Tabla 4. Apariciones de pronombres átonos de objeto directo en el habla de $\mathbf{H 4}$

\begin{tabular}{|c|c|c|c|c|c|c|c|c|}
\hline \multicolumn{3}{|c|}{ LOS } & \multicolumn{3}{|c|}{ LO } & \multicolumn{3}{|c|}{ LA } \\
\hline \multicolumn{3}{|c|}{$(1 \mathrm{de} 45)$} & \multicolumn{3}{|c|}{ (43 de 45) } & \multicolumn{3}{|c|}{$(1 \mathrm{de} 45)$} \\
\hline & Referentes & Abs & & Referentes & Abs & & Referentes & Abs \\
\hline \multirow{6}{*}{ Disc. } & Masc. sing. & 0 & \multirow{6}{*}{ Disc. } & Masc. sing. & 0 & \multirow{6}{*}{ Disc. } & Masc. sing. & 0 \\
\hline & Masc. pl. & 0 & & Masc. pl. & 0 & & Masc. pl. & 0 \\
\hline & Fem. sing. & 0 & & Fem. sing. & 18 & & Fem. sing. & 0 \\
\hline & Fem. pl. & 1 & & Fem. pl. & 6 & & Fem. pl. & 0 \\
\hline & Múltiple & 0 & & Múltiple & 1 & & Múltiple & 0 \\
\hline & Total & 1 & & Total & 25 & & Total & 0 \\
\hline \multirow{6}{*}{ Conc. } & Masc. sing. & 0 & \multirow{6}{*}{ Conc. } & Masc. sing. & 18 & \multirow{6}{*}{ Conc. } & Masc. sing. & 0 \\
\hline & Masc. pl. & 0 & & Masc. pl. & 0 & & Masc. pl. & 0 \\
\hline & Fem. sing. & 0 & & Fem. sing. & 0 & & Fem. sing. & 1 \\
\hline & Fem. pl. & 0 & & Fem. pl. & 0 & & Fem. pl. & 0 \\
\hline & Múltiple & 0 & & Múltiple & 0 & & Múltiple & 0 \\
\hline & Total & 0 & & Total & 18 & & Total & 1 \\
\hline
\end{tabular}

Tal y como se puede observar, el clítico lo se realiza en un 43 ocasiones frente a la y los, que ocurren en tan solo 1 ocasión cada una: la en concordancia con un referente femenino plural, y los en discordancia con el referente masculino plural. Para lo se registran 18/18 formas concordantes con referente masculino singular y 25 formas discordantes, estas últimas distribuidas en 18/18 discordancias con referentes femeninos singulares; 6/6, con referentes femeninos plurales, y 1/1 con referentes múltiples. Como vemos, la mayor cantidad de discordancias del pronombre lo ocurren con referentes femeninos singulares.

37. El hermano al verlo se asustó (al hermano). ConC.

38. La serpiente de una vez lo mordió al muchacho. ConC.

39. Él lo veía (a la muchacha). DisC.

40. Al verlo a esa muchacha. Disc.

41. Se lo llevaba (al papá y a la mamá). DisC.

42. Uno lo utilizaba (las partes básicas). Disc.

43. Algunas palabras no me lo sé en español. Disc.

44. La oración la dice en guaymí. CoNC.

45. Puedo detallarlos (las plantas). Disc.

Para el entrevistado en cuestión, no se identificó ninguna omisión de objeto directo.

\section{$2.5 \quad H 5$}

Consideramos que H5 entra en la categoría de bilingüe simétrico, pues el propio hablante indica que su comprensión y producción tanto en guaymí como en español es muy equilibrada. 


\subsubsection{Conteo de pronombres átonos de objeto directo en el habla de H5}

En H5, se identificaron los pronombres átonos $l o(s)$ y la en función de objeto directo, cuyos porcentajes de aparición se pueden observar en la siguiente tabla:

Tabla 5. Apariciones de pronombres átonos de objeto directo en el habla de $\mathbf{H 5}$

\begin{tabular}{|c|c|c|c|c|c|c|c|c|}
\hline \multicolumn{3}{|c|}{ LOS } & \multicolumn{3}{|c|}{ LO } & \multicolumn{3}{|c|}{ LA } \\
\hline \multicolumn{3}{|c|}{$(1 \mathrm{de} 75)$} & \multicolumn{3}{|c|}{ (73 de 75$)$} & \multicolumn{3}{|c|}{$(1 \mathrm{de} 75)$} \\
\hline & Referentes & Abs & & Referentes & Abs & & Referentes & Abs \\
\hline \multirow{5}{*}{ Disc. } & Masc. sing. & 0 & \multirow{5}{*}{ Disc. } & Masc. sing. & 0 & \multirow{5}{*}{ Disc. } & Masc. sing. & 0 \\
\hline & Masc. pl. & 0 & & Masc. pl. & 12 & & Masc. pl. & 0 \\
\hline & Fem. sing. & 0 & & Fem. sing. & 12 & & Fem. sing. & 0 \\
\hline & Fem. pl. & 0 & & Fem. pl. & 5 & & Fem. pl. & 0 \\
\hline & Total & 0 & & Total & 29 & & Total & 0 \\
\hline \multirow{5}{*}{ Conc. } & Masc. sing. & 0 & \multirow{5}{*}{ Conc. } & Masc. sing. & 44 & \multirow{5}{*}{ Conc. } & Masc. sing. & 0 \\
\hline & Masc. pl. & 1 & & Masc. pl. & 0 & & Masc. pl. & 0 \\
\hline & Fem. sing. & 0 & & Fem. sing. & 0 & & Fem. sing. & 1 \\
\hline & Fem. pl. & 0 & & Fem. pl. & 0 & & Fem. pl. & 0 \\
\hline & Total & 1 & & Total & 44 & & Total & 1 \\
\hline
\end{tabular}

El clítico de objeto directo lo se presenta en 73 ocasiones, cuyas apariciones de concordancias y discordancias se distribuyen en 44 y 29 casos, respectivamente. Se registran $44 / 44$ concordancias con referentes masculinos singulares. En cambio, las discordancias de lo ocurren con referentes masculinos plurales en 12/12 ocasiones; con referentes femeninos singulares, en 12/12, y con referentes femeninos plurales, en 5/5. Así las cosas, la cantidad más alta de discordancias de lo (y de hecho idéntica) aparece en sintagmas correferenciales tanto femeninos singulares como masculinos singulares.

Por otra parte, la forma plural los y la forma femenina la ocurre en 1 cada una, en concordancia con los sintagmas referidos: masculino plural y femenino singular, respectivamente. Al comparar el empleo de la variante singular lo con el de la variante plural los y la, se observa que la primera resulta ser mucho más usada que las otras dos formas.

46. Castiguémoslo (al muchacho). Conc.

47. El oro se lo trajeron. Conc.

48. Lo volcó (a la bruja). Disc.

49. ¿Para qué lo mandó? (a los cuatro). Disc.

50. Esos perros lo amarró. Disc.

51. Comenzaron cortarlo diferente (a las serpientes). Disc.

52. Los vi allá (a los demás). Conc.

53. La están viendo a la otra. CONC.

Para el hablante en cuestión, se registra un único caso de clítico elidido (54).

54. Él se quedó viendo $\emptyset$ (al camarón) 


\subsection{H6}

Se trata de un bilingüe consecutivo con el español como lengua dominante en cuanto a su capacidad de hablar y bilingüe simétrico de acuerdo con su capacidad de comprensión oral.

\subsubsection{Conteo de pronombres átonos de objeto directo en el habla de H6}

En el habla de H6, se identificó el uso de los clíticos la y lo, cuyos valores numéricos de aparición se observan en la siguiente tabla:

Tabla 6. Apariciones de pronombres átonos de objeto directo en el habla de H6

\begin{tabular}{|c|c|c|c|c|c|}
\hline \multicolumn{3}{|c|}{ LO } & \multicolumn{3}{|c|}{ LA } \\
\hline \multicolumn{3}{|c|}{ (20 de 23 ) } & \multicolumn{3}{|c|}{ (20 de 23$)$} \\
\hline & Referentes & Abs & & Referentes & Abs \\
\hline \multirow{5}{*}{ Disc. } & Masc. sing. & 0 & \multirow{5}{*}{ Disc. } & Masc. sing. & 2 \\
\hline & Masc. pl. & 0 & & Masc. pl. & 0 \\
\hline & Fem. sing. & 2 & & Fem. sing. & 0 \\
\hline & Fem. pl. & 0 & & Fem. pl. & 0 \\
\hline & Total & 2 & & Total & 2 \\
\hline \multirow{5}{*}{ Conc. } & Masc. sing. & 18 & \multirow{5}{*}{ Conc. } & Masc. sing. & 0 \\
\hline & Masc. pl. & 0 & & Masc. pl. & 0 \\
\hline & Fem. sing. & 0 & & Fem. sing. & 1 \\
\hline & Fem. pl. & 0 & & Fem. pl. & 0 \\
\hline & Total & 18 & & Total & 1 \\
\hline
\end{tabular}

El pronombre átono lo se presenta en 20 ocasiones; en cambio, la realización femenina la ocurre en 3 casos. También, hemos registrado para lo 18/18 casos de concordancias con referentes masculinos singulares, y $2 / 2$ casos de discordancias con referentes femeninos singulares y 18/18 casos de concordancias, mientras que para la, las discordancias ocurren en 2/2 casos con referentes masculinos singulares, en tanto se identificó $1 / 1$ concordancia con referente femenino singular.

En H6, la cantidad de discordancias de lo con sintagmas femeninos singulares se ve disminuida frente a las cantidades registradas para otros entrevistados en esta categoría. Otros datos que se pueden inferir de la Tabla 6 son: primero, que la cantidad de discordancias de $l o$ y la coincide para sendos pronombres (2/2 casos para cada uno) y segundo, que la discordancia en ambas formas se limita al género, pues sus respectivos sintagmas correferenciales poseen rasgo singular.

55. Que se lo iba a comer (al tepezcuintle). ConC.

56. Un muchacho todo lo habla en español. Conc.

57. A veces no lo cuidan (la casa). Disc.

58. Que tenía que cuidarla (al tepezcuintle). Disc.

59. Para ponerla (la piedra). CONC.

Para H6, no se identificó ningún caso de omisión. De hecho, él y H4 son los únicos hablantes de la muestra en los que no ocurre este fenómeno. 


\subsection{HHC (Hablante hispanocostarricense de control)}

La situación de HSC es muy distinta a la de los guaymíes remitidos anteriormente, pues se trata de un hablante monolingüe del español que vive en una zona aledaña al territorio indígena en el que habitan los miembros de la muestra principal. Estos datos nos permitirán obtener un patrón de usos pronominales contrastable con el de los guaymíes, a fin de tener mayores indicios de cuáles fenómenos son producto del contacto entre el guaymí y el español, y cuáles otros pueden originarse sin que exista dicho contacto.

En la Tabla 7, se puede observar la cantidad de ocasiones en las que el hablante hispanocostarricense utiliza cada clítico:

Tabla 7. Apariciones de pronombres átonos de tercera persona de objeto directo según las propiedades gramaticales en HHC

\begin{tabular}{|l|c|c|}
\hline & \multicolumn{2}{|c|}{ Objeto directo } \\
\cline { 2 - 3 } & Clíticos & Abs \\
\hline $\begin{array}{l}\text { Referente masculino singular } \\
\text { sin reduplicación }\end{array}$ & lo & 48 \\
\hline $\begin{array}{l}\text { Referente masculino plural sin } \\
\text { reduplicación }\end{array}$ & los & 6 \\
\hline $\begin{array}{l}\text { Referente masculino singular } \\
\text { con reduplicación }\end{array}$ & lo & 9 \\
\hline $\begin{array}{l}\text { Referente masculino plural con } \\
\text { reduplicación }\end{array}$ & los & 1 \\
\hline $\begin{array}{l}\text { Referente femenino singular sin } \\
\text { reduplicación }\end{array}$ & la & 30 \\
\hline $\begin{array}{l}\text { Referente femenino plural sin } \\
\text { reduplicación }\end{array}$ & las & 6 \\
\hline $\begin{array}{l}\text { Referente femenino singular } \\
\text { con reduplicación }\end{array}$ & la & 8 \\
\hline $\begin{array}{l}\text { Referente femenino plural con } \\
\text { reduplicación }\end{array}$ & las & 2 \\
\hline \multicolumn{2}{|c|}{ Total } & 110 \\
\hline
\end{tabular}

HSC utiliza las formas $l o(s)$ y $l a(s)$, ya sea para referirse a sintagmas femeninos singulares (57 y 58) o plurales (59 y 60), o masculinos singulares $(61$ y 62) o plurales (63 y $64)$. En ambos casos, los sintagmas pueden aparecer reduplicados $(57,59,61$ y 63$)$ o no (58, $60,62$ y 64$)$.

\section{La matrícula ahí la tengo}

58. Ella quería llevársela para Heredia (a la hija)

59. Todas se las contestaba

60. No las tiene (las cosas)

61. Todo se lo fue memorizando

62. Lo pusieron manos así al carro (al muchacho)

63. Todos los trabajos gracias a Dios los sabe hacer

64. Yo se los limpio bien barato (los zapatos) 
En contraste con las tendencias de empleo por parte de los guaymíes, los valores numéricos de la Tabla 7 indican la aparición de formas de objeto directo marcadas con género femenino singular y plural (la y las) y masculino plural (los). Tampoco debemos perder de vista que todas las formas identificadas en el corpus de HHC concuerdan con su referente, comportamiento muy distinto al observado en el habla de los guaymíes, cuyos usos pronominales manifiestan tendencias variadas de concordancia o discordancia gramatical.

\subsection{Explicación de los resultados cuantitativos}

La tabulación de datos permite interpretar que, en el español de los guaymíes entrevistados, existe una clara tendencia a utilizar el clítico lo de objeto directo en discordancia con los sintagmas correferenciales por encima de las formas femeninas $l a(s)$ y el masculino plural los.

Para $\mathrm{H} 1, \mathrm{H} 2, \mathrm{H} 3$ y H4, la categoría de referente femenino singular presenta la mayor cantidad de discordancias de $l o$, frente al resto de categorías expuestas en cada una de las Tablas 1, 2, 3 y 5. En el discurso de H5, se observó que las discordancias de lo expresan sus frecuencias más altas en las categorías de femenino singular y masculino plural. Por otra parte, las únicas dos discordancias de lo identificadas en el discurso de H6 ocurren en la categoría de referentes femeninos singulares, mientras que dos discordancias de la se presentan en la categoría de masculino singular (este último es el único hablante que manifiesta discordancia para el pronombre femenino singular). Con todo, inferimos que los usos discordantes de lo se extienden en mayor proporción a los referentes con género femenino y número singular, y con menor proporción a otras categorías con referentes masculinos plurales o femeninos plurales. Es así como las discordancias gramaticales de lo vendrían a reflejar la neutralización de género y número a favor de este pronombre.

En consecuencia, todas las observaciones permiten diferenciar entre el sistema pronominal de los guaymíes y el de $\mathrm{HHC}$, quien emplea $l a(s)$ y $l o(s)$ estableciendo la concordancia gramatical del español sin contacto. Sumado al uso predominante de $l o$, en el discurso oral de $\mathrm{H} 1, \mathrm{H} 2, \mathrm{H} 3, \mathrm{H} 4$ y $\mathrm{H} 5$ identificamos omisiones de objeto directo, fenómeno que no ocurre en $\mathrm{H} 6, \mathrm{H} 4 \mathrm{y} \mathrm{HHC}$.

Los resultados en torno a las discordancias contrastan con el sistema pronominal etimológico registrado para el español costarricense sin contacto (Agüero, 2009; QuesadaPacheco, 2009), en el cual se hace distinción entre el dativo marcado con $l e(s)$ y el acusativo marcado con $l o(s)$ y $l a(s)$. De hecho, HHC ejemplifica adecuadamente el comportamiento de este tipo de sistema.

\section{Análisis cualitativo}

En seguida, se describe la simplificación del sistema átono de tercera persona por medio de escalas; en el segundo, se contrastan las propiedades gramaticales de los objetos directo en guaymí y español costarricense sin contacto con otra lengua, y en los restantes, se estudian los fenómenos de la neutralización a favor de lo como objeto directo y las propiedades gramaticales del guaymí que podrían estar influyendo en esta neutralización.

\subsection{Procesos de simplificación}

En la Tabla 8, se muestran las características de los subsistemas idiolectales y sus diversos grados de simplificación con base en la aparición de clíticos registrada en el apartado 2. 
Además, el término "subsistema" se refiere a los resultados obtenidos a partir de cada idiolecto que se reflejan en los estadios de la simplificación misma:

Tabla 8. Organización del sistema pronominal átono de tercera persona de objeto directo en la muestra poblacional

\begin{tabular}{|l|l|}
\hline Hablantes & Pronombres de objeto directo \\
\hline H1 & lo,, , la \\
\hline H2 & lo, $\varnothing$ \\
\hline H3 & lo(s), $\varnothing$, la \\
\hline H4 & lo(s), la \\
\hline H5 & lo(s), $\varnothing$, la \\
\hline H6 & lo, la \\
\hline HSC & lo(s), la(s) \\
\hline
\end{tabular}

Por lo que se refiere al objeto directo, la tabla anterior muestra una conducta del sistema pronominal que se puede explicar asumiendo la coexistencia de diferentes subsistemas pronominales con diferentes grados de simplificación (ver Tabla 9) incluso en un mismo hablante. Como se aprecia en la Tabla 9, en el primer grado de simplificación, no se codifica la marca del plural femenino, aunque se trata de usos de la concordante, tal y como H3, H4 y H5 lo manifiestan. El grado de simplificación siguiente neutraliza la distinción de número en lo y la, como se puede observar en $\mathrm{H} 1$ y H6. El resultado concreto es la coexistencia en un mismo hablante de patrones concordantes y discordantes en diferentes grados y cantidades; es decir, que en ninguna variedad idiolectal de los guaymíes existe un sistema puro.

Un grado de simplificación aún mayor se manifiesta en $\mathrm{H} 2$, pues para este hablante, lo constituye la única forma. Además, la simplificación máxima ocurre con la ausencia de los clíticos, la cual se marca con el cero fonético $(\varnothing)$, tal y como se ilustra en los hablantes $\mathrm{H} 1, \mathrm{H} 2$, H3 y H5. Asimismo, hemos podido constatar que el comportamiento pronominal registrado para los guaymíes contrasta en gran medida con el de HHC, en cuyo sistema de clíticos (el sistema pronominal etimológico) se hace distinción sistemática de género y número: $l o(s)$ y $l a(s)$. La discordancia vendría a ser una consecuencia estructural del grado de neutralización a favor de un pronombre específico, lo cual va parejo con el grado de simplificación en el proceso de reestructuración del sistema.

A manera de síntesis, se propone la siguiente escala de simplificación para el sistema de clíticos de objeto directo. Cada número representa un estadio del proceso de simplificación.

Tabla 9. Escala de simplificación para el sistema de pronombres átonos de tercera persona de objeto directo

\begin{tabular}{|l|l|l|l|}
\hline $\begin{array}{l}\text { 1. Sistema etimológico } \\
\rightarrow\end{array}$ & $\begin{array}{l}2 . l o(s) \\
\rightarrow\end{array}$ & $\begin{array}{l}3 . l o \\
\rightarrow\end{array}$ & $4 . \varnothing$ \\
\hline
\end{tabular}

El primer estadio (correspondiente al sistema etimológico) es el único que presenta marcación de plural en el femenino (65). Como vimos en la Tabla 8, este sistema solo se expresa en el hablante hispanocostarricense (HHC), en cuyo corpus se registra el uso del clítico las, a diferencia del de los guaymíes, en cuya utilización de pronombres hay una ausencia sistemática de la forma femenina plural las, debido a que, para los referentes femeninos y plurales, suele ser utilizado el clítico lo (de ahí que se pueda hablar de neutralización de género). 


\section{(65) Las cosas las entendió de lo más bien (HHC)}

Desde otra perspectiva, en el sistema pronominal que aparece en el habla de los guaymíes H3 y H5, coexiste el primer estadio con el segundo y cuarto estadio, pues además del uso concordante de la con el referente femenino y singular (66 y 69) (primer estadio) y la presencia del clítico lo discordante sin marca de plural (69 y 70) (segundo estadio), ocurren omisiones del objeto directo (68 y 71), las cuales, si bien resultan ser poco significativas en términos cuantitativos (ver apartado 2), vienen a constituir posibilidades estructurales presentes en los corpus de estos hablantes.

66. No sé si usted la conoce la planta (H3)

67. Yo trato de entenderlo (las dos culturas) (H3)

68. Pero llegó descubrirØ (las plantas para esa enfermedad) (H3)

69. La están viendo (a la otra) (H5)

70. Lo iba a dejar a las hermanas (H5)

71. Él se quedó viendo Ø (al camarón) (H5)

Por su parte, el subsistema de objeto directo reflejado en H4 se ubicaría en el primer (72) y segundo estadio (73) de la escala, pues presenta la concordante y lo discordante (en género y número):

\section{La oración la dice en ngäbe $(\mathrm{H} 4)$}

73. Él lo utilizaba (las partes básicas) (H4)

Para H1, el subsistema oscila entre el primer, tercer y cuarto estadio, dado que emite las formas lo (74) y la (75), además de omisiones (76). Se considera que el clítico lo en este hablante pertenece al tercer estadio y no al segundo, porque no se registra con marca de plural:

74. Usted lo ve morenitos a todos (H1)

75. Ponerla a germinar (la semilla) (H1)

76. Ellos más bien no Ø mostraba (interés) (H1)

Por su parte, el primer y tercer estadio se manifiesta en el individuo H6, quien emplea las formas singulares la concordante (77) y lo discordante sin marca de plural (78), y en cuyo subsistema no se registran omisiones.

\section{Para ponerla ahí (la piedra) (H6)}

\section{A veces no lo cuidan (la casa) (H6)}

No obstante, más arriba indicamos que H6 utiliza la para referirse tanto a sintagmas masculinos como a femeninos, mientras que $\mathrm{H} 1$ solamente utiliza este clítico para referirse a sintagmas femeninos. Dicho de otra manera, si bien el sistema pronominal de ambos hablantes está integrado por los mismos pronombres, la opción de neutralización puede ser diferente, ya que el objeto directo en H6 vendría a neutralizarse a favor de lo y a veces a favor de la (79), hecho lingüístico no identificado en $\mathrm{H} 1$, ni tampoco en otros hablantes de la muestra poblacional.

\section{El tigre no fue a sacarla (al tepezcuintle) (H6)}

Por otra parte, el uso de lo en $\mathrm{H} 2$ muestra la simplificación máxima para ambos sistemas de clíticos, en donde las formas masculinas y femeninas, plurales y singulares, se neutralizan en lo (80). Esta simplificación ubica a $\mathrm{H} 2$ en el tercer estadio. Al mismo tiempo, 
este fenómeno de simplificación a favor de lo coexiste con el de omisión de clíticos (81) perteneciente al cuarto estadio.

80. $Y$ así yo quiero que la persona lo respete en raza indígena $(\mathrm{H} 2)$

81. Mi papá yo no Ø vi (H2)

Lo dicho hasta aquí supone que existen grados de simplificación, a partir de los cuales hemos podido hipotetizar cómo se organiza el subsistema de pronombres átonos de tercera persona para cada variedad idiolectal de los entrevistados. Recordemos que en este tipo de simplificación actúa el mecanismo de convergencia lingüística (ver apartado 1.1), el cual promueve que se dé una aproximación las características estructurales de los clíticos en español a la estructura del guaymí, y por lo tanto, se reinterpreten las funciones de estos pronombres átonos. Para comprender cómo opera este mecanismo en el comportamiento y organización del sistema pronominal en estudio, mostraremos las propiedades gramaticales del guaymí que podrían estar motivando la simplificación de este sistema pronominal en el español de los guaymíes.

\subsection{Influjo del guaymí en la simplificación del sistema pronominal descrito}

En lo que corresponde al guaymí, esta lengua chibcha hace distinción formal entre las relaciones gramaticales de sujeto, objeto directo e indirecto El sintagma nominal en función de objeto directo no posee ningún tipo de marcación morfológica y ocupa la posición rígida preverbal ya sea que se exprese como sintagma nominal (82) o como pronombre personal (83) (Quesada-Pacheco, 2008a).

82. Felipe ñaka tugwe ngwen kämigu-e

Felipe NEG ratón blanco matar-PRS

'Felipe no mata ratones blancos' (Murillo-Miranda, 2016)

\section{Pedro gwe niara kämi-ri}

Pedro ERG 3SG matar-P.REC

'Pedro lo mató (a él)'14

El objeto directo en guaymí también puede ser expresado como pronombre o anáfora cero. Con respecto a este fenómeno, obsérvese el siguiente pasaje de un texto narrativo tomado de Quesada-Pacheco (2008b), en el cual ocurre el fenómeno susodicho:

84a. Ngäbe jäme matanibi nügue ben ja bätä, ${ }^{15}$

Gente tranquilo aterrizar-REC llegar-IND con REF cerca,

'Estando la gente descuidada chocaba con la gente'

84b. jännibira niguen kuin kwe kwetara

cargar-REC-COMP ir-IND arriba para+que comer-FIN

'inmediatamente [la] alzaba para comer [la]'

84c. ngäbe kira negä jumu küse te

gente venir-REC -COMP desaparecer-IND ave+fénix mano en.

'y la gente fue desapareciendo en manos del ave fénix'

84d. Ägwä miga janani sukia ie tre.

Ojo poner-IIR ir- REM chamán DAT PL

'fueron al suquia para que [la] ojeara' 


\section{4e. Sukia bätäkä ie ñan nibi nuane}

chamán diferente DAT no quedar-REC hacer-IND.

'a diferentes suquias les fue imposible eliminar[la]' (Quesada-Pacheco, 2008b)

Tal y como observamos, en la oración (84b) se elide el sintagma nominal ngäbe 'gente' en función de objeto directo, el cual se había mencionado en (84a), mientras que en la oración (84e) se elide jumu 'ave fenix', objeto directo que había sido mencionado previamente en (84c).

Según Krohn (2014), para que el objeto directo se pronominalice o se omita, es necesario que su referente sea altamente accesible, pues tanto el uso de los pronombres niara o kwe como la anáfora cero solo aportan información de la tercera persona gramatical, lo que implica que no categoricen rasgos morfosintácticos de género y número del sintagma nominal al cual se refieren. Por tanto, ambas estrategias referenciales se encuentran en un mismo estatus funcional para objetos directos en el guaymí. Por lo dicho, tanto el pronombre de tercera persona como la anáfora constituyen unidades sin contenido semántico en contraste con la expresión léxica que aportan únicamente información sobre la tercera persona gramatical.

Por su parte, el español (sin contacto) hablado en Costa Rica se caracteriza por el empleo de pronombres átonos de objeto directo de acuerdo con el sistema etimológico que distingue género en la marcación de caso acusativo por medio de las formas $l o(s)(83)$ y $l a(s)$ (84) ejemplos tomados de Quesada-Pacheco (2009).

\section{María encontró a Juan y a Pedro y los saludó}

\section{Marta fue a casa de su abuela; cuando la saludó le entregó un regalo}

Al contrastar las propiedades de objeto directo del guaymí y el español, observamos las siguientes características: en ambas lenguas es posible codificar los objetos directos mediante sustantivos, pronombres personales y otras clases de palabra como cuantificadores, indefinidos, etc., y en ambas lenguas, es posible recurrir a la anáfora cero (Ø). Una diferencia relevante es que el guaymí carece de un sistema pronominal átono como el del español. Desde el punto de vista de la función de los pronombres átonos (es decir, mantener activos referentes en el transcurso de la enunciación).

Otra diferencia importante es en cuanto al género y la marcación de número. Según Quesada-Pacheco (2008b) y Murillo-Miranda (2016), no se registra ninguna marcación de género en el sustantivo, y la marcación de número, que puede calificarse como optativa, se realiza en los sustantivos y pronombres mediante el uso de un morfema de plural que se manifiesta en las formas -tre $\sim$-dre (para humanos) o -grä (para animales y cosas). QuesadaPacheco (2008b) registra la marcación de plural -tre, colocado a la derecha de la palabra (pronombre u otro), como en (85). Asimismo, se restringe a no aparecer cuando se ha utilizado en oraciones anteriores (86). En contraste, en el español hay todo un sistema flexivo que es obligatorio, incluso en el sistema pronominal átono (87). Este último ejemplo fue tomado del RAE (2010).

\section{Mä jämi kwe-tre llamar ngw-en-däri kwe-tre}

2 SG falta 3-PL llamar preguntar-PRS- preguntar 3-PL

'Le falta llamarlos para preguntarles'

86. mun kwata ürügrö ere kwe-tre, ngäre merire gwe

cera corteza recoger-REC mucho 3-PL gente mujer ERG

mun kö riri

cera cuerda cocer- REC

'Recogieron mucha cera, las mujeres cocinaron la sustancia de la miel' 


\section{Las eligieron a ellas}

Es evidente, entonces, que el guaymí posee un sistema de flexión nominal reducido comparado con el del español y que ambas lenguas coinciden (parcialmente) y convergen en los mecanismos de codificación de las relaciones gramaticales involucradas. Con todo, el contraste entre las propiedades gramaticales de los objetos en guaymí y español costarricense (sin contacto) debe servir para explicar las particularidades del sistema pronominal átono de tercera persona en el español de los guaymíes como resultado del contacto con el guaymí.

\section{Conclusiones}

A partir de la cuantificación de fenómenos relativos al sistema pronominal átono en estudio, se observó que la cantidad de apariciones registradas para el pronombre lo supera la cantidad de omisiones de objeto directo. Dentro de las apariciones de objeto directo, el pronombre $l o$ en discordancia con los sintagmas correferenciales prima sobre las formas $l a(s)$ y los. La tendencia al uso mayoritario de lo viene a constituir un resultado estructural de la neutralización de los rasgos de género y número en la variedad del español hablada por los guaymíes entrevistados. Así pues, esta neutralización de rasgos conlleva a la simplificación del sistema pronominal, lo cual es sintomático del cambio indirecto. De hecho, esta simplificación pareciera ser la diferencia más importante entre el sistema pronominal identificado en el español guaymí y el español costarricense sin contacto lingüístico.

En todos los entrevistados se da la neutralización a favor del pronombre lo de objeto directo, cuyos rasgos gramaticales etimológicos de género y número son masculino y singular, respectivamente. También observamos muchos casos en los que el clítico lo se refiere a sintagmas femeninos, lo que responde a un patrón sobre el cambio inducido por contacto en el sistema pronominal átono de tercera persona: dentro del conjunto de clíticos que codifican caso acusativo, se pierde la distinción de género a favor del pronombre menos marcado lo y se refleja un sistema más simple de objeto directo (Palacios-Alcaine, 2011).

Finalmente, la neutralización a favor de lo de objeto directo es resultado de la pérdida de distinciones de concordancia gramatical que no existe en la lengua de contacto (el guaymí), debido al mecanismo de convergencia lingüística.

\section{Notas}

1. En el libro Historia de la Lengua Española en Costa Rica de Quesada-Pacheco (2009) se hace referencia en un apartado al español de un hablante chorotega de principios del siglo XX (consúltese de la página 467 a la 477$)$.

2. En el apartado 1., la etiqueta "español sin contacto" también se puede referir al español sin contacto con otras lenguas.

3. Palacios-Alcaine (2011) explica los tipos de bilingüismo a partir de contactos lingüísticos entre el español y lenguas indígenas como el quichua y el guaraní. La nomenclatura "lenguas indígenas" se ha utilizado tal y como se hace en el artículo de Palacios.

4. Cada hablante declaró su tipo de bilingüismo en una entrevista semiestructurada (Marín, 2017). En el presente artículo, no se profundiza en las correlaciones que puedan haber entre bilingüismo, edad y sistema pronominal átono de objeto directo. Para ampliar en detalles, consúltese Marín (2017).

5. Los guaymíes llaman sulia al hispanocostarricense.

6. Este tipo de entrevista se denomina "historia sociolingüística" y se define como una información que "[...] nos puede dar indicios [...] sobre la competencia bilingüe esperable del sujeto [...]” (Sánchez-López y Rodríguez-De Tembleque, 1997, p. 82). 
7. La Nueva Gramática de la lengua Española (RAE, 2010) presenta los fenómenos generales del español (sin contacto) de modo más descriptivo que en sus versiones anteriores.

8. Las oraciones agramaticales *Vieron a ella, ${ }^{*}$ El periódico compra mi hijo y *El que desee fumar, puede hacer; son adaptaciones nuestras.

9. Las letras en negrita señalan el clítico y su sintagma correferencial. Algunos de dichos sintagmas se encuentran entre paréntesis porque corresponden a formas no reduplicadas. La reduplicación no ha sido un aspecto tratado en este artículo.

10. ¿Mä töa ño? se traduce al español como el saludo '¿Cómo está usted?'.

11. El ñuture es una planta conocida también como 'disciplina de monja' (Caesalpinia gilliesii).

12. El término 'referente múltiple' significa que un mismo clítico hace referencia a una serie de sintagmas: p. ej. a mi mamá, a mi abuelo y a mi abuela yo sí lo vi.

13. El término 'muri(s)' se refiere a buglé(s).

14. Ejemplo elicitado por la autora.

15. Quesada-Pacheco (2008b) no presenta la segmentación morfológica en guaymí.

\section{Bibliografía}

Agüero-Chaves, A. (2009). El español de Costa Rica. San José: Editorial de la Universidad de Costa Rica.

Aleza, M. y Enguita, J. M. (2010). La lengua española en América: normas y usos actuales. València: Universitat de València.

Appel, R. y Muysken, P. (1996). Bilingüismo y contacto de lenguas. Barcelona: Ariel.

Constenla-Umaña, A. (1991). Las lenguas del Área Íntermedia: Introducción a su estudio areal. San José: EUCR.

Constenla-Umaña, A. (2002). Algunos aspectos lingüísticos y socioculturales de la influencia de las lenguas indígenas en las variedades americanas del español. http://lanicutexas. edu/.project/etext/llilas/cilla/constenla.pdf [Consulta 13 de julio de 2015].

Kaufman, T. y Thomason, S. (1988). Language contact, creolization and genetic linguistics, Berkeley: University of California Press.

Krohn-Stensrud, H. (2014). Análisis cuantitativo del mantenimiento de la referencia anafórica en ngäbe (guaymí). Revista Káñina. 38 (1), 203-216.

Lininger-Ross, B. (1991). El español de los bribris: su estructura interna. (Tesis doctoral). Universidad de Costa Rica.

Matras, Y. (2009). Language Contact. Cambridge: Cambridge University Press.

Marín-Esquivel, R.(2017). El sistema pronominal del español hablado por noves costarricenses desde la perspectiva del contacto de lenguas. (Tesis de Maestría). Universidad de Costa Rica.

Martín-Crespo, C. y Salamanca-Castro, A. B. (2007). El muestreo en la investigación cualitativa. Nure Investigación, 27.

Moreno-Fernández, F. (1998). Principios de Sociolingüística y sociología del lenguaje. Barcelona: Ariel Letras.

Murillo-Miranda, J. M. (2016). Gramática de la lengua guaymí. Dialecto de Costa Rica: una descripción urgente. Saarbrücken: EAE. 
Palacios-Alcaine, A. (2002). El sistema pronominal átono del español paraguayo: un caso de contacto de lenguas. Por J. Calvo (Coord.). Teoría y práctica del contacto: el español en América en el candelero (123-143). Madrid: Vervuet- Iberoamericana.

Palacios-Alcaine, A. (2004). Aspectos teóricos y metodológicos del contacto de lenguas: El sistema pronominal del español en áreas de contacto con lenguas amerindias. Por I. Neumann, K. Zimmermann y V. Noll (Coords). El español en América: aspectos teóricos, particularidades, contactos. (63-92). https://dialnet.unirioja.es/servlet/ articulo?codigo=2353896 [Consulta 22 de octubre de 2016].

Palacios-Alcaine, A. (2005). El sistema pronominal del español ecuatoriano: un caso de cambio lingüístico inducido por el contacto. Por C. Chevonenu y Y. Lastra (Eds.). Dinámica lingüística de las lenguas en contacto. (357-376). Hermosillo: Universidad de Sonora.

Palacios-Alcaine, A. (2005). Situaciones de contacto lingüístico en Hispanoamérica: español y lenguas amerindias. Por L. Girón (Coord.). Actas del VI Congreso Internacional de Historia de la Lengua española. (1- 15). Madrid: Arco Libros.

Palacios-Alcaine, A. (2007). ¿Son compatibles los cambios inducidos por contacto y las tendencias internas al sistema? Por M. Schrader-Kniffki y L. Morgenthaler-García (Eds.). Lenguas en interacción: Entre historia, contacto y política. Ensayos en homenaje a Klaus Zimmermann. (259-279). Frankfurt/M, Vervuert.

Palacios-Alcaine, A. (2008). El español en América. Contactos lingüísticos en Hispanoamérica. Barcelona: Ariel.

Palacios-Alcaine, A. (2010). Algunas reflexiones en torno a la lingüística del contacto. ¿Existe el préstamo estructural? Revista Internacional de Lingüística Iberoamericana (RILI). 8 (15), 33-55.

Palacios-Alcaine, A. (2011). Nuevas perspectivas en el estudio del cambio inducido por contacto: hacia un modelo dinámico del contacto de lenguas. Revista de Lenguas Modernas. 38, 17-36.

Portilla-Chaves, M. (1986). Un caso de muerte de lenguas: el térraba. Estudios de Lingüística Chibcha. 5, 97-246.

Quesada-Pacheco, M. A. (2008). América Central. Por A. Palacios (Coord.). El español en América. Contactos lingüísticos en Hispanoamérica (57-69). Barcelona: Ariel.

Quesada-Pacheco, M. A. (2008). Gramática de la lengua guaymí (ngäbe). Munchen: Lincom Europa.

Quesada-Pacheco, M. A. (2009). Historia de la lengua española en Costa Rica. San José: Editorial de Costa Rica.

Sánchez-Avendaño, C. (2008). El uso de "le" por "les" en el español costarricense: de la cohesión a la morfologización. Revista Internacional de Lingüística Iberoamericana. 1 (11), 111-127.

Sánchez-Avendaño, C. (2012). El papel de la escuela en el desplazamiento y en la conservación de la lengua malecu. Revista Educación. 36 (1), 25-43.

Sánchez-Avendaño, C. (2013). Lenguas en peligro de Costa Rica: vitalidad, documentación y descripción. Káñina. 37 (1), 219-250. 
Sánchez-Avendaño, C. (2015). El sistema pronominal átono de tercera persona en el español hablado por los malecus de Costa Rica. CLAP. 61, 79-103.

Sánchez-López, P. y Rodríguez-De Tembleque, R. (1997). El bilingüismo. Bases para la intervención psicológica. Madrid: Síntesis, S. A.

Silva-Corvalán, C. (2001). Sociolingüística y pragmática del español. Washington: Georgetown University Press.

Real Academia de la Lengua Española. (2010). Manual de la nueva gramática de la lengua española. Madrid: Asociación de Academias de la Lengua Española.

Thomason, S. (2001). Language Contact. Edinburgh: University Press.

\section{Abreviaturas}

SG singular

NOM nominativo

PRF perfecto

CFL clasificador

P.REC pasado reciente

PRS presente

P. REM pasado remoto

DAT dativo

NEG negación

PL plural

ERG ergativo

REM remoto

PF perfecto

ITER marcador de iteratividad

PRP propositivo

REC reciente

IND tiempo indefinido

REF marcador de reflexividad o reciprocidad

COMP marcador de completividad

FIN marcador de finalidad

IIR indefinido irrealis

FUT futuro

F. REM futuro remoto

RAE Real Academia Española

Conc. Concordancia

Disc. Discordancia 